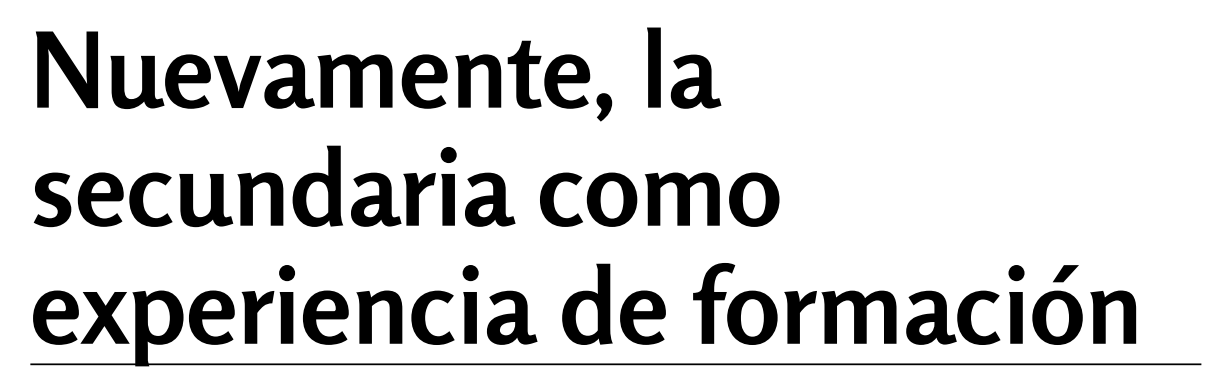





\title{
Sentidos y finalidades de la experiencia escolar de estudiantes de escuelas secundarias de Resistencia y Corrientes
}

\author{
(1) Patricia Malena Delgado \\ Universidad Nacional del Nordeste, Argentina \\ patridelgadow@gmail.com \\ Cinthia Denise Amud \\ Universidad Nacional del Nordeste, Argentina \\ deniamud@gmail.com \\ Jorge Samuel Salas \\ Universidad Nacional del Nordeste, Argentina \\ jorgesamuelsalas@gmail.com
}

\begin{abstract}
Resumen
En este trabajo nos proponemos describir las visiones que construyen los/as estudiantes en torno a la escuela secundaria, contrastando sus valoraciones y expectativas con sus percepciones acerca de lo que la escuela les brinda en cuanto a trabajo con el conocimiento y la preparación para continuar estudios superiores, para el ingreso al mundo laboral y para la formación ciudadana y en valores. En función de ello analizamos resultados obtenidos de la aplicación de cuestionarios semi-estructurados a estudiantes que se encontraban terminando sus estudios secundarios en cuatro escuelas públicas de gestión estatal, dos de la ciudad de Corrientes y dos de Resistencia (Chaco). Complementamos el análisis examinando resultados de cinco grupos de discusión, en los que participaron estudiantes que estaban cursando los últimos años de estudio en dos de las escuelas donde inicialmente se aplicaron las encuestas.El análisis de cómo perciben y valoran los y las estudiantes la formación recibida en la escuela secundaria en relación consus finalidades nos ha permitido explorar la construcción del sentido que asume la experiencia escolar para ellos/as.
\end{abstract}

Palabras clave: Educación secundaria, experiencia escolar secundaria, finalidades de la escuela secundaria, estudiantes secundarios 


\title{
Senses and purposes of the school experience of high school students of Resistencia and Corrientes
}

\begin{abstract}
In this paper we propose to describe the visions that students build around high school, contrasting their assessments and expectations with their perceptions about what the school offers them in terms of work with knowledge and preparation to continue higher studies for entry into the world of work and citizenship training and values. To do this, we analyzed results obtained from the application of semi-structured questionnaires to students who were finishing secondary school in four public schools, two in the city of Corrientes and two in Resistencia (Chaco). We complemented the analysis by examining the results of five discussion groups, in which students who were studying the last years of study, participated in two of the schools where the surveys were initially applied. Studying how students perceive and value the training received in high school in relation to their purposes, has allowed us to explore the construction of the sense that the school experience assumes for them.
\end{abstract}

Keywords: Secondary education, high school experience, high school purposes, high school students

\section{Introducción}

El objetivo de nuestro trabajo es describir las visiones que construyen los/as estudiantes en torno a la escuela secundaria, con referencia a sus sentidos y finalidades. En particular, nos interesa analizar el modo en que estos/as perciben y valoran la formación recibida en relación con la preparación para continuar estudios superiores, la formación para el trabajo y la formación en actitudes y valores necesarios para la convivencia y el ejercicio de la ciudadanía.

Asimismo, mediante la comparación de las respuestas en las cuatro instituciones públicas de las ciudades de Corrientes y Resistencia, que conforman la muestra, procuramos reconocer similitudes y diferencias en las visiones de los estudiantes, considerando las características de los contextos institucionales y las condiciones socioeconómicas de los/as alumnos/as y sus familias.

Para ello, examinamos datos que corresponden a investigaciones enmarcadas en una línea de trabajo desarrollada por el Grupo de Estudios sobre Escuela Secundaria $(\text { GEES })^{1}$. Particularmente retomamos hallazgos emergentes de un trabajo de tesis doctoral (de Cinthia Amud) ${ }^{2} y$ de una beca de investigación de pregrado(realizada por Jorge Salas) ${ }^{3}$.

Organizamos el artículo en cuatro apartados. En primer lugar, precisamos los principales supuestos teóricos que enmarcan nuestra investigación. En segundo lugar, presentamos algunas precisiones metodológicas respecto a cómo se delimitó la muestra

1El proyecto denominado "La experiencia escolar en la escuela secundaria. Perspectivas y participación de actores educativos para la acción transformadora", acreditado por la SGCyT- UNNE, dirigido por María Delfina Veiravé y codirigido por Patricia Delgado.

2Tesis desarrollada en el marco del Proyecto de Investigación mencionado, y en laCarrera de Doctorado en Ciencias de la Educación de la UNC. Fue financiado por una Beca de Iniciación de la SGCyT-UNNE y otra beca de CONICET.

3En el marco de una Beca de Pregrado de SGCyT-UNNE. 
y cuáles fueron los instrumentos de recolección y análisis de datos utilizados; además realizamos una breve caracterización del grupo de estudiantes que participaron en el estudio y de los establecimientos a los que pertenecen. En tercer lugar, presentamos el análisis de las valoraciones que realizan los/as estudiantes sobre la formación recibida con relación a su grado de adecuación a las finalidades de la escuela secundaria. Por último,exponemos en las Conclusiones una síntesis de los principales hallazgos que derivan del estudio realizado.

\section{Algunas precisiones teóricas acerca de los sentidos de la experiencia escolar}

Para comprender las percepciones y valoraciones de los/as estudiantes en torno a la formación recibida y su adecuación a las finalidades de la escuela secundaria, partimos del supuesto de que estas concepciones son construcciones sociales, de carácter situacional y relacional, que se definen a partir de las experiencias escolares vividas por los/as estudiantes y de expectativas y proyectos de vida futuros.

Dicho supuesto deriva del modo en que abordamos el concepto de experiencia escolar, integrando perspectivas teóricas que consideramos complementarias. Así, desde el campo pedagógico, la experiencia es definida como una construcción subjetiva individual (Larrosa, 2004; Contreras y Pérez de Lara, 2010) porcuanto refiere las vivencias que construye cotidianamente un sujeto en un escenario institucional y en interacción con otros/as. En este sentido, presume un acontecimiento exterior pero vivido o padecido por alguien a través de un relato de palabras, significados y representaciones (Madonni, 2014).

Las experiencias escolares comprenden modos de hacer, conocer y relacionarse, que adoptan características específicas e influyen en la construcción de sentidos asignados al trabajo escolar y al tránsito por la escuela secundaria (Guzmán Gómez y Saucedo Ramos, 2015). En este marco, el término sentidos alude a "motivos para", que remiten a un estado de situación deseable y a intenciones que orientan la acción humana desde el punto de vista del actor (Schutz, 1974, en Andrade, 2010).

En consonancia con estos análisis, Rockwell $(1995,2006)$ sostiene que el contenido de la experiencia escolar se transmite mediante el conjunto de prácticas escolares cotidianas que configuran el contexto formativo real de los/as estudiantes, constituido por la transmisión del conocimiento, la organización de actividades de enseñanza y los vínculos institucionales que sustentan el proceso escolar (Rockwell, 1995). Por lo tanto, dichas prácticas condicionan el carácter y el sentido de lo que es posible aprender en la escuela, promoviendo en los sujetos la apropiación de diversos conocimientos, valores y formas de vivir (Rockwell, 2006).

En esa diversidad de contextos formativos puede identificarse la desigual distribución de capitales y condiciones materiales y simbólicas que caracteriza a nuestras sociedades (Llomovatte y Kaplan, 2012), origen de las desigualdades escolares y sociales reflejadas en la configuración fragmentada del sistema educativo y en dinámicas de diferenciación de circuitos escolares según nivel de calidad educativa y sector social que atienden (Tiramonti, 2011; Tiramonti y Montes, 2009).

Desde una perspectiva sociológica, las consideraciones anteriores pueden articularse en torno al concepto de "experiencia escolar", definido por Dubet y Martuccelli (1998) como el trabajo mediante el cual actores individuales y colectivos combinan las diversas lógicas de acción que estructuran el mundo escolar y logran socializarse y constituirse 
como sujetos. Los autores distinguen tres tipos de lógicas de acción: la lógica de integración al sistema escolar, que se corresponde con su función de socialización; la lógica estratégica, que responde a la función de distribución de competencias con utilidad social, asociada a mecanismos de movilidad social; y la lógica de subjetivación, promovida por la escuela,que en correspondencia con su función educativaalude a la capacidad de crítica, de individualización y acción autónoma del sujeto. Estas lógicas -vale aclararlo- subyacen al sentido que tiene la experiencia escolar para los sujetos que la construyen cotidianamente.

Las perspectivas teóricas expuestas consideran las vivencias y sentidos que asume la escuela para los individuos reconociendo la capacidad de los sujetos de re-construir significados, a través de su puesta en acción o mediante la narración de sus experiencias (Guzmán Gómez y Saucedo Ramos, 2015). De igual modo, estos enfoques hacen énfasis en el carácter situado de las experiencias escolares, construidas en contextos institucionales particulares atravesados por condiciones y transformaciones sociales, culturales y políticas que inciden en los procesos de subjetivación y socialización.

Con abordajes similares, otros estudios realizados enlaArgentina exploraron las visiones que construyen los/as estudiantes sobre la escuela secundaria. En particular retomamos los aportes de Dussel, Brito y Núñez (2007), quienes entre 2005 y 2008 realizaron un estudio de alcance nacionaldonde indagaron las percepciones y expectativas de estudiantes y profesores sobre la escuela secundaria. Una vía para examinar los sentidos que la educación adquiere para los/as jóvenes fue el análisis de los aprendizajes más valorados durante su tránsito por la escuela. En este punto, advirtieron algunas cuestiones comunes ydiferencias significativas según sector social. Entre las semejanzas, se observó un alto porcentaje de alumnos/as de diferentes niveles socioeconómicos que valoran las relaciones o disposiciones sociales como los aprendizajes más importantes que ofrece la escuela (socializar con otros, aprender a cumplir normas), así como valores de responsabilidad, respeto y solidaridad. La escuela es significada como espacio de reconocimiento de derechos, desarrollo personal, construcción identitaria yun ámbito que orienta, organiza, ordena y estructura a los sujetos. Entre las diferencias, encontraron que los/as jóvenes de sectores medios y altos enfatizan la construcción de vínculos con los profesores en torno a la transmisión de conocimiento en las materias, mientras que sus pares de niveles socioeconómicos bajos priorizan relaciones más horizontales con los docentes, construidas en torno al cuidado y la contenciónpor sobre la transmisión de saberes.

En otra investigación, Llinás (2009) examinó los sentidos que los/as estudiantes otorgan a su experiencia escolar. En cuanto a las visiones que tienen de sus escuelas, encontró en general una valoración positiva: ellos/as esperan ser formados en términos de contenidos, de normas, y que la escuela se constituya en un espacio de socialización entre pares. Complementando estas imágenes positivas, surgieron críticas vinculadas con el bajo, desigual o poco exigente nivel educativo; en tal dirección, señalaron la dificultad que esto genera al momento de ingresar a la universidad. En este sentido, la demanda es que la escuela se constituya en un espacio que habilite la transmisión de conocimientos en una experiencia educativa más intensa.

Asimismo la autora mencionada identificó tres grupos de sentidos de la experiencia escolar que no difieren sustancialmente según jurisdicciones, sectores sociales y modalidades. En primer lugar, un grupo de respuestas que pueden agruparse como "sentidos tradicionales", referidos a continuar estudiando, trabajar, adquirir conocimientos e integrarse socialmente. El más recurrente fue el de continuar los estudios, que aparece como la principal motivación para asistir a la escuela secundaria. Sin embargo, algunas diferencias según sector social permiten reconocer expectativas "fuertes", dado que cuentan con apoyos de las escuelas para que se realicen, y otras descritas como 
"atenuadas":lasnosostenidas poracciones de las escuelas o las familias y que, según los/ as estudiantes, condicionan sus posibilidades de concreción. En segundo lugar, identificó el grupo conformado por tres "sentidos híbridos" (Llinás, 2009): obtener el título de la escuela secundaria, "lograr ser alguien" y la formación en valores. En el primer caso, los/as estudiantes son conscientes de la devaluación de esta credencial educativa, sin embargo consideran que la finalización del secundario constituye un piso básico para acceder al empleo. Además, la posibilidad de transitar la escuela secundaria para "ser alguien" evidencia que la promesa de progreso social que tradicionalmente porta la escuela secundaria continúa presente en las representaciones de los/as alumnos/as demarcando identidades y futuros, aunque cobra nuevos matices en el contexto social actual. El último sentido, de formación en valores y atribuido a la escuela, difiere según las características de la institución y los valores que busca transmitir.

La autora identificóun último grupo de sentidos, menos frecuentes, denominados "emergentes", los cuales ponen de manifiesto que la experiencia escolar se asocia a un presente productivo, con relación a estar en la escuela, pasarla bien, y en algunos casos también asociado al gusto por aprender.

La autora concluye que los sentidos atribuidos a la escuela son mediados por la cultura y el imaginario social, que se relacionan tanto con sus expectativas de futuro e integración social como con el tiempo presente que transcurre mientras transitan en ella. En nuestra investigación arribamos a conclusiones semejantes a partir del análisis de las percepciones y valoraciones de los/as estudiantes acerca de la formación recibida en función de los propósitos o finalidades del nivel.

\section{Algunas precisiones sobre la estrategia metodológica}

Al examinar los diferentes niveles y dimensiones de análisis que conforman la problemática estudiada, el abordaje metodológico combinó distintas estrategiasde construcción de información:cuantitativas y cualitativas.

En el año 2015 se aplicaron cuestionarios semi-estructurados a jóvenes que estaban finalizando sus estudios secundarios en cuatro escuelas públicas de gestión estatal, dos de la ciudad de Corrientes (en adelante $\mathrm{C} 1$ y C2) y dos de la ciudad de Resistencia (en adelante R1 y R2), en ambos casos situadas en zonas céntricas (R2 y C2) y periféricas (R1 y C1). En las escuelas $\mathrm{C} 1$ y C2, se seleccionó una submuestra del $40 \%$ del total de estudiantes del último año, definida mediante muestreo probabilístico estratificado, tomando todas las divisiones como estratos, al interior de los cuales se realizó la selección aleatoria de casos. Del mismo modo se procedió para conformar la submuestra de estudiantes de R2, conformada por alumnos de los dos últimos años de estudio. Solamente en R1 se encuestó a la totalidad de estudiantes de los dos últimos años, por tener una matrícula menor. De este modo, la muestra quedó constituida por un total de 434 estudiantes: 97 de R1; 191 de R2; 65 de C1; y 81 de C2. La aplicación del cuestionario permitió relevar datos sobre variables sociodemográficas de los sujetos, así como sus percepciones sobre finalidades de la educación secundaria y prácticas de enseñanza y aprendizaje en el ámbito escolar. El análisis estadístico de los datos se realizó con el programa SPSS.

Durante el año 2016 se realizaron cinco grupos de discusión, en los que participaron 41 estudiantes de $\mathrm{C} 1$ y R1 que se hallaban cursando los dos últimos años de estudio. Mediante este instrumento se pretendió ampliar y profundizar en aspectos de la experiencia escolar, que fueron centrales desde el comienzo de la investigación, y sobre temas emergentes identificados en el primer momento de trabajo de campo. Para ello se 
conformó una muestra intencional siguiendo criterios de heterogeneidad (edad, sexo, trayectorias escolares, desempeño escolar, divisiones en las que cursan y orientaciones del plan de estudio). Para el proceso de análisis e interpretación de los datos obtenidos en los grupos de discusión, se utilizó el software NVivo, el cual facilitó el trabajo de codificación e identificación de temas y sus relaciones.

Cabe aclarar que la selección de las escuelas no pretendió abarcar una muestra representativa, sino seleccionar algunos escenarios posibles de construcción de experiencias estudiantiles en escuelas públicas de Chaco y Corrientes. Si bien se trata de escuelas que trabajan con diversidad de estudiantes en cuanto a sus condiciones materiales y trayectorias educativas, y que tienen historias institucionales y tradiciones distintas, encontramos ciertas características semejantes conforme a la ubicación de los establecimientos.

Las escuelas C2 y R2se encuentran ubicadas en zonas céntricas de las ciudades y gozan de cierto prestigio social en la calidad educativa que ofrecen. Ambas tienen un número elevado de matrícula y gran demandaen los primeros años, la cual excede las vacantes disponibles. Por esta razón, en la escuela R2 se toman exámenes de ingreso, ${ }^{4}$ mientras que en C2 las vacantes se cubren estableciendoun orden de mérito según el promedio de calificaciones obtenidas en las escuelas primarias de las cuales provienen los/as estudiantes.

Por su lado, a las instituciones $\mathrm{C} 1$ y R1, ubicadas en zonas periféricas, asistenestudiantes de sectores populares. Son "escuelas de barrio", imagen que emerge en las entrevistas a sus directivos y en los grupos de discusión. Surgieron como escuelas de comercio, y esa orientación se conserva en parte en la actualidad en los nuevos planes de estudio. Mientras que en $\mathrm{C} 1$ la cantidad de estudiantes resulta numerosa, la matrícula de R1 es mucho menor.

En cuanto a los perfiles socioeducativos de los/as estudiantes, las escuelas ubicadas en zonas periféricas ( $\mathrm{R} 1$ y $\mathrm{C} 1$ ) presentan mayor número de alumnos/as que trabajan y mayores porcentajes de sobreedad y repitencia. Estos datos están en consonancia con indicadores de trayectoria escolar de los subsistemas de nivel secundario de ambas provincias. En Chaco, en 2015 el promedio de estudiantes con sobreedad fue del 35,2\%, guarismo que se eleva en las instituciones de gestión estatal hasta alcanzar el $38 \%$. Por otra parte, en Corrientes el 43,5\% de los estudiantes secundarios cursa con sobreedad, cifra que se eleva al $48 \%$ en las instituciones de gestión estatal, mientras que los encuestados en las escuelas de zonas céntricas (R2 y C2) tienen niveles de repitencia bajos y escasa sobreedad. En cuanto a su situación laboral, en R2 solo el 8\% trabaja, mientras que en C2 lo haceel $20 \%$.

Respecto al perfil del grupo familiar, ${ }^{5}$ en las escuelas ubicadas en zonas periféricas hay una alta proporción de padres y madres que no alcanzaron a terminar el nivel secundario y tienen empleos que no requieren estudios o mayores titulaciones. Los padres en su mayoría se desempeñan en algún oficio (albañil, plomero, electricista, etc.) o como empleados de comercios, fábricas e industrias, mientras que la mayoría de las madres trabajan como empleadas domésticas.

En las escuelas céntricas, en cambio, se puede observar una alta proporción de padres y madres que terminaron el nivel medio y accedieron o terminaron alguna carrera de 
educación superior. Esto se evidencia también en los puestos de trabajo que ocupan, pues se desempeñan en cargos de dirección o gestión de empresas o instituciones públicas, como profesionales independientes o en profesiones que requieren titulación secundaria o superior.

Esta diversidad de contextos socioterritoriales e institucionales nos ha permitido comparar y reconocer continuidades y variaciones en las percepciones y valoraciones de los y las estudiantes que participaron en el estudio.

\section{Percepciones y valoraciones de estudiantes sobre la formación recibida en relación con las finalidades de la escuela secundaria}

Examinando las miradas que tienen los/as estudiantes sobre las finalidades del nivel medio y cómo evalúan la formación recibida, en este apartado retomamos las preguntas del formulario de encuesta:

¿Crees que la educación que te ofrecen en la escuela secundaria te prepara de manera adecuada para continuar estudiando una carrera universitaria o terciaria?

¿Crees que la educación que te ofrecen en la escuela te prepara adecuadamente para ingresar al mundo laboral?

¿Crees que la educación que te ofrecen en la escuela te prepara de manera adecuada para la vida social en democracia, es decir, para ser un ciudadano/a responsable, consciente de tus derechos y obligaciones?

Además de contestar "sí", "no" o la opción "no sabe/no contesta", se solicitó a los/as estudiantes que fundamentaran sus respuestas. También incluimos en este apartado hallazgos que surgen de los grupos de discusión, en particular del análisis de respuestas a las preguntas: para qué asistís a la escuela, qué aprendés y qué te aporta la escuela secundaria. A continuación, presentamos los resultados obtenidos así como el análisis de los criterios utilizados por los/as estudiantes en sus valoraciones.

\section{a) La formación para continuar estudios superiores}

Respecto de esta finalidad, el 65\% de la muestra respondió que se lo estaba preparando en forma adecuada, mientras que el $28 \%$ sostuvo que no y el $7 \%$ no respondió a la pregunta. Veamos cómo se distribuyen las respuestas según los establecimientos:

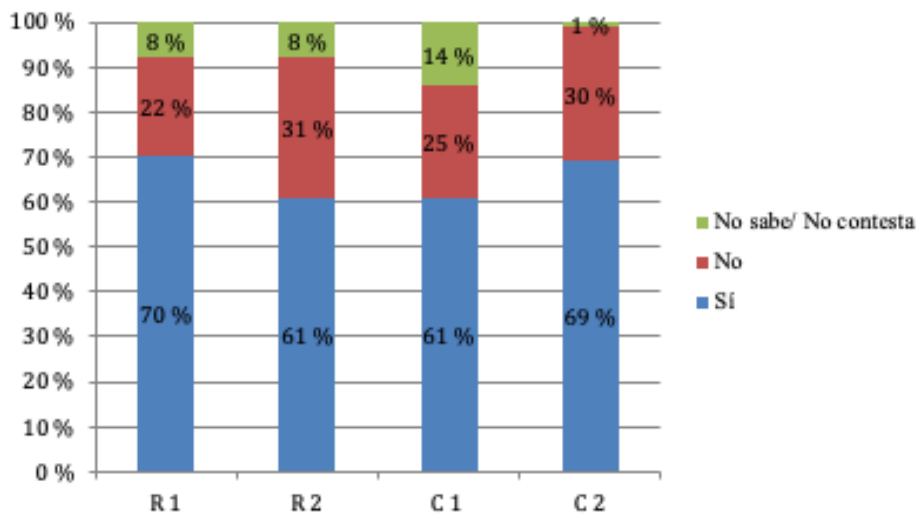

Gráfico1. Distribución de los estudiantes por institución y si consideran que su establecimiento forma adecuadamente para continuar estudios superiores 
Más del 60\% de los/as encuestados/as en las cuatro escuelas consideró que se les estaba ofreciendo una formación adecuada para continuar estudios superiores, terciarios o universitarios, siendo levemente superiores los porcentajes en R1 y C2. No se advierten grandes variaciones entre escuelas en las respuestas negativas. Sin embargo, encontramos diferencias en los modos de fundamentar las opiniones de acuerdo con la ubicación del establecimiento al que pertenecen.

Para los/as estudiantes de las escuelas $\mathrm{R} 1$ y C1, las enseñanzas y orientaciones que reciben de sus profesores/as les permiten estar mejor preparados para elegir una carrera y continuar estudiando, porque contagian su entusiasmo y pasión por las disciplinas que enseñan y de ese modo los inspiran a estudiarlas. Se trata de un aporte desde lo motivacional, como lo ilustra la cita:

Porque algunos profesores te inspiran a estudiar las carreras que ellos estudiaron, por el entusiasmo con el que te enseñan. (Alumna de R1)

Asimismo, valoran a docentes que explican bien los contenidos, haciendo referencia a la enseñanza de conocimientos necesarios para continuar estudios superiores:

Porque te orientan bien en todas las clases o materias que tenemos y los profesores son muy buenos en su trabajo, explican bien todo. (Alumna de $\mathrm{C}_{1}$ )

En sus opiniones, el acceso a la educación superior se presenta como una condición necesaria para acceder a mejores trabajos y así adquirir un nuevo estatus o posición social, que se condensa en la expresión "ser alguien en la vida", como lo ilustran estas citas:

...te preparan bien para que puedas seguir una carrera, ser alguien el día de mañana. (Alumna de R1)

Porque creo que para eso es la escuela, prepararnos para continuar estudiando una carrera para poder tener un trabajo digno en el futuro. (Alumno de R1)

La escuela secundaria se representa como una oportunidad de progreso social, lo que puede entenderse si se considera que en las familias de estos/as estudiantes un gran porcentaje de padres y madres no concluyeron sus estudios secundarios y se desempeñan en ocupaciones de baja calificación. En este sentido, un alumno expresa:"me gustaría aprovechar la oportunidad que mis padres no tuvieron y quiero darles esa satisfacción" (Alumno de R1).

Por otra parte, examinando las respuestas afirmativas de las escuelas R2 y C2, sus argumentos se centran más en el nivel de preparación y exigencia académica de la institución. Particularmente en R2 se valora la propuesta curricular del bachillerato como "una buena base" donde se trabajan todos los contenidos necesarios para el ingreso a cualquier carrera de educación superior:

Yo creo que sí, es una herramienta fundamental para ingresar a cualquier carrera. Este colegio por ejemplo tiene el bachillerato que para mí es mejor [...]. Porque la mayoría de los chicos no están seguros sobre lo que quieren estudiar y es mejor que den un poquito de todo. (Alumna de R2)

Asimismo señalan aspectos vinculados a las formas de trabajo con el conocimiento que proponen sus docentes y los niveles de exigencia en las evaluaciones:

Porque el nivel de exigencia es muy similar al grado o nivel de exigencia que me darán en una universidad. (Alumno de $\mathrm{C}_{2}$ ) 
...en el último (año) de secundario te preparan bastante, te enseñan técnicas de estudio, a hacer informes, algunos profesores nos hacen trabajar con libros más avanzados para que nos acostumbremos y nos sea más fácil entenderlos. (Alumna de $\mathrm{C}_{2}$ )

Comparando estas opiniones con las de estudiantes de C1 y R1, se advierte una mayor centralidad del trabajo con los contenidos disciplinares como base para la adquisición de conocimientos en la educación superior. Se mencionan con frecuencia aspectos relativos a las formas de trabajo con el conocimiento, valorizando el aprendizaje de técnicas de estudio, el acceso a materiales de lectura semejantes a los que estudiarán en la universidad, o los niveles de exigencia en las evaluaciones. Encontramos hallazgos similares en la investigación de Dussel, Brito y Núñez (2007), quienes detectaron en jóvenes de sectores medios y altos un mayor énfasis en la construcción de vínculos con los profesores en torno a la transmisión de conocimiento en las materias, mientras que sus pares de niveles socioeconómicos bajos priorizaban relaciones con los docentes construidas en torno al cuidado y la contención por sobre la transmisión de saberes.

Este criterio de valoración, que pone énfasis en la centralidad del trabajo con los contenidos disciplinares y los niveles de exigencia académica, fundamenta en los cuatro establecimientos las respuestas de quienes sostienen que la formación recibida no los está preparando para continuar estudios superiores:

... no, porque las carreras universitarias son más exigentes y avanzadas académicamente. (Alumna de $\mathrm{C}_{1}$ )

No, porque siempre año tras año vuelven a dar lo básico y repetir lo del año anterior. (Alumno de R1)

Aparece la idea de que la universidad implica un nivel de dificultad y de exigencia mayor, un modo de trabajo al que no están acostumbrados, ya sea porque los contenidos no son trabajados con profundidad y amplitud, o por la baja exigencia académica.

En los grupos de discusión que se realizaron en C1 y R1, advertimos similitudes en los argumentos de quienes están disconformes con la preparación para continuar estudios superiores. Conviene aclarar que entre quienes expresaron su intención de continuar estudiando, son pocos los/as que pretenden ingresar al ámbito universitario,y son ellos/ as quienes enfatizan las críticas respecto de la formación recibida. Recordemos que en la investigación de Llinás (2009) también surgieron críticas vinculadas con el bajo, desigual o poco exigente nivel educativo, que señalaron la dificultad que esto genera al momento de ingresar a la universidad.

Además, en R1 los/as estudiantes mencionan como problema la falta de asistencia regular a clases por paros docentes continuos, ${ }^{6}$ ya que no se dictan los contenidos necesarios que deberían ser aprendidos para el ingreso a la educación superior: “... porque la mayoría de las veces hay paro o jornadas que nos dejan pocos días de clase” (Alumna de R1).

A partir del análisis de estas percepciones y valoraciones de los/as estudiantes, nos preguntamos cómo construyen sus imágenes sobre la vida estudiantil en la educación superior, considerando que estas constituyen las referencias de las cualesdisponen para evaluar la formación recibida.

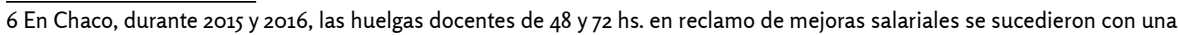
frecuencia semanal o quincenal. Esta situación de conflicto continuó en 2017, registrándoseg2 días de paro, cifra que superó los 83 días de clase (Fuente: Diario Norte, noticia del 12/02/2018). Vale aclarar que no en todos los establecimientos educativos hubo,entre sus docentes,el mismo nivel de adhesión a los paros. 
Retomando los aportes de Rockwell (1995), si el contenido de la experiencia escolar se transmite mediante el conjunto de prácticas escolares cotidianas que configuran el contexto formativo real de los/as estudiantes, y dichas prácticas condicionan el carácter y el sentido de lo que es posible aprender en la escuela, nos preguntamos: ¿Les anticipa la escuela algo de lo que será su vida como estudiante en ese nivel educativo? ¿Se reconoce en ella la intencionalidad de preparar para ese horizonte de futuro y facilitar así la transición? Y por otra parte, ¿cómo influyen los niveles de estudio y las actividades laborales que realizan sus padres y madres en esas visiones y expectativas?

Aun cuando no tenemos datos suficientes para caracterizar las prácticas escolares cotidianas de las escuelas seleccionadas, pues no hemos realizado estudio de casos, podemos proponer algunas conjeturas para responder a estas preguntas.

Las escuelas C2 y R2 se definen como instituciones que preparan para la continuidad de estudios superiores. Esta finalidad forma parte de su identidad institucional. Puede que por esa razón, y por el perfil socioeducativo de sus alumnos/as, se desarrollen prácticas formativas como las mencionadas por ellos/as cuando valoran positivamente la formación recibida. Pero consideramos también que en las percepciones, valoraciones y expectativas estudiantiles se reproducen las representaciones y expectativas familiares sobre los estudios superiores, sus exigencias académicas y el tipo de formación propedéutica que esperan de la escuela secundaria. Desde ese imaginario evalúan en forma positiva o negativa la formación recibida. De allí que los/as estudiantes de C2 y R2, cuyos padres y madres tienen niveles educativos más altos y se desempeñan en empleos más calificados, tengan mayores referencias en su ámbito familiar y social para representarse las características y exigencias de los estudios superiores, y otorguen por ello más importancia al trabajo con los contenidos disciplinares y los niveles de exigencia académica.

En cambio, R1 y C1, desde su creación como escuelas comerciales, se orientaron más a una formación de carácter práctico e instrumental que posibilitara una inserción laboral inmediata. Por esa orientación, y considerando las características socioeducativas de estudiantes con quienes trabajan, puede que en las prácticas formativas que proponen no esté tan presente la intencionalidad de formar para continuar estudios superiores, sino que sus estudiantes terminen la escuela secundaria y estén en condiciones de conseguir un empleo. También en estos casos las representaciones y expectativas familiares influirían en el modo que los/as estudiantes evalúan la formación recibida y se proyectan a futuro. El hecho de que muchos padres y madres no hayan concluido estudios secundarios, o accedido al nivel superior, podría limitar su visión respecto de los requerimientos de ese ámbito académico y la importancia de trabajar contenidos disciplinares como base para acceder a tal formación. También podría contribuir a explicar que tengan expectativas más bajas o que consideren poco factible acceder a la universidad o a un terciario para continuar estudiando.

\section{b) La formación para ingresar al mundo del empleo}

El 55\% de los/as encuestados/as respondió que la formación recibida es adecuada para la inserción laboral, mientras que el $27 \%$ respondió que no lo es y el $18 \%$ no contestó la pregunta. No obstante, los valores promedio ocultan diferencias que se presentan entre los establecimientos, como lo muestra el Gráfico 2. 


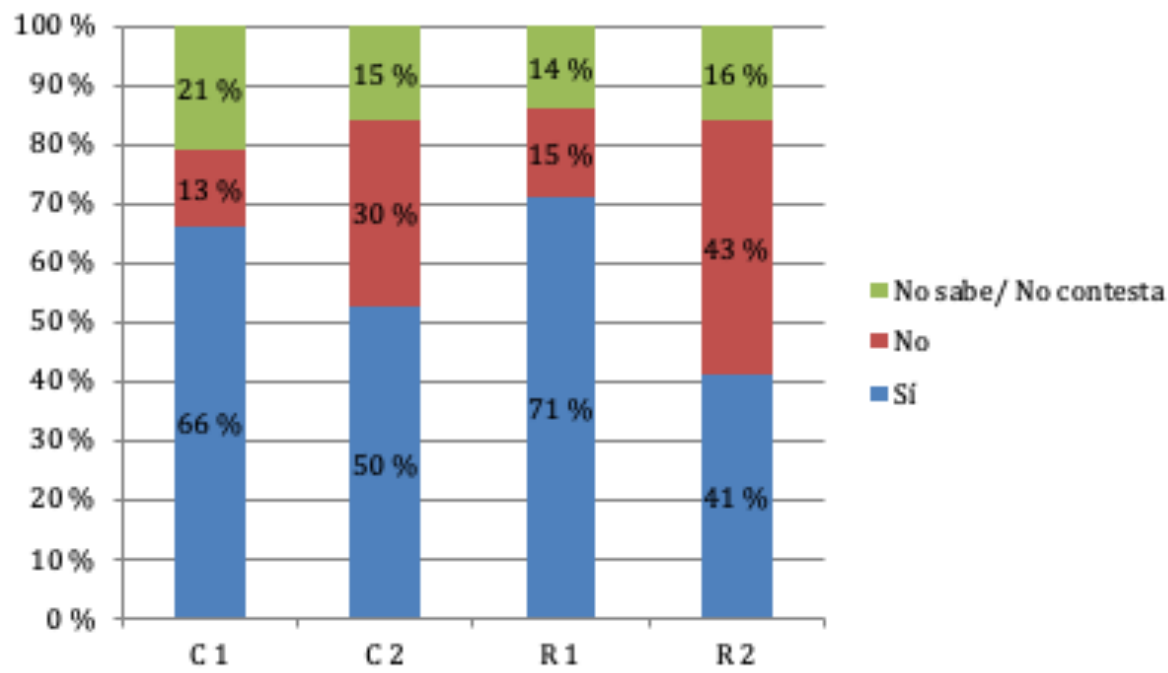

Gráfico 2. Distribución de los estudiantes por institución y si consideran que su establecimiento forma adecuadamente para ingresar al mundo laboral

En este gráfico se advierte que las/os estudiantes de las instituciones localizadas en áreas periféricas ( $\mathrm{R} 1$ y $\mathrm{C} 1)$ dan más respuestas afirmativas que en los establecimientos de zonas céntricas ( $\mathrm{R} 2$ y C2). Puede que estas diferencias se deban, por un lado, a que en C1 y R1 existe un mayor número de estudiantes que trabajan y estudian (35\% y 28\%, respectivamente). $\mathrm{Al}$ respecto, un estudiante de $\mathrm{R} 1$ señalaba: "ya he tenido experiencia laboral y puse en práctica la enseñanza de la escuela y me ha ayudado mucho".

Por otra parte, en las razones que justifican las respuestas de $\mathrm{R} 1$ y $\mathrm{C} 1$ reaparece la idea de la escuela secundaria como oportunidad de progreso social, en tanto el título secundario permite acceder a mejores trabajos. En palabras de una alumna de R1: "porque, hoy en día, si no terminaste el secundario no tenés un buen trabajo".En respuestas como esta se evidencia que no necesariamente los/as estudiantes están considerando la posibilidad de continuar estudios superiores para acceder a un mejor empleo.

El trabajo con los grupos de discusión permitió captar matices en esta visión de la escuela secundaria como oportunidad de progreso social. Así, en los dos grupos de R1 surgió la imagen de la escuela como obligación necesaria y como medio para obtener un título que habilite la inserción laboral futura. El objetivo de obtener el diploma constituye el principal fundamento para permanecer en la escuela. Las miradas de este grupo de estudiantes están teñidas de críticas hacia un conjunto de condiciones materiales, pedagógicas y curriculares que producen una progresiva pérdida de interés hacia el trabajo escolar. En coincidencia con lo que observaron Maldonado, Servetto y Molina (2015) en escuelas secundarias de la provincia de Córdoba, para estos/as jóvenes la escuela puede convertirse en un espacio cargado de desencuentros debido a reiteradas ausencias de estudiantes y docentes, a la cantidad de horas libres y a la pérdida de días de clases, lo que dificulta la continuidad de los procesos de aprendizaje.

A pesar de su disconformidad, los/as estudiantes manifiestan que "aguantan" esta situación por la necesidad de finalizar el trayecto escolar y conseguir un título que los habilite para el ingreso al ámbito laboral y obtener así una remuneración para la propia subsistencia. Tal como observaron Montesinos, Sinisi y Schoo en escuelas secundarias del conurbano bonaerense, para estos/as jóvenes la educación secundaria se configura como una "obligación necesaria a la que le otorgan un valor instrumental anclado exclusivamente en la expectativa de que la posesión del título mejoraría su inserción laboral y social a futuro" (Montesinos, Sinisi y Schoo, 2009: 5). 
En cambio, en los grupos de discusión de C1, los/as estudiantes valoran la obtención del título secundario como condición necesaria para acceder a un empleo, pero refieren además a la adquisición efectiva de conocimientos y competencias para el desempeño laboral. La adquisición del diploma no es el único criterio de valoración que consideran, pues recuperan aprendizajes que logran desarrollar a través de ciertas actividades escolares.

En dos de los grupos de $\mathrm{C} 1$ particularmente se valoran espacios curriculares como Proyectos y Gestión de Microemprendimientos, en los que desarrollan actividades como talleres, con el objetivo de aprender a elaborar y comercializar diferentes productos en forma grupal y colaborativa. También en las encuestas, los/as estudiantes de C1 mencionan la importancia de la orientación "comercial" que les permite desarrollar actividades como proyectos o microemprendimientos, en coincidencia con lo que surgió en los grupos de discusión.

Como aprendizajes necesarios para la inserción laboral, en las respuestas al cuestionario se mencionan saberes prácticos, hábitos, pautas de comportamiento y actitudes, como lo ilustran los testimonios que siguen:

Porque te orientan en cómo formar tu propio emprendimiento, ser responsables en todos los trabajos y sobre todo siempre la buena presentación. (Alumna de $\mathrm{C}_{1}$ )

Porque los profesores nos enderezan en cómo saber y utilizar el respeto, la solidaridad, la tolerancia que son aquellos valores que nos ayudarán a ingresar a un buen mundo laboral. (Alumna de $\mathrm{C}_{2}$ )

Estudiantes que evalúan positivamente la formación recibida mencionan que sus escuelas les permiten desarrollar actitudes y valores como la responsabilidad, la puntualidad y la dedicación, cualidades que definen a un "buen trabajador". En el caso de C2 y R2, las respuestasserefieren además al aprendizaje de conocimientos básicos necesarios para continuar estudios superiores y así poder ingresar al mercado laboral. Sus estudiantes sostienen que finalizar la educación media no es condición suficiente para acceder a puestos de trabajo, ya que es necesario un conocimiento más especializado que se desarrolla en la educación superior.

En cuanto a las respuestas negativas, que son superiores en $\mathrm{C} 2$ y $\mathrm{R} 2$, estas remiten principalmente a la falta de trabajo sobre ciertos conocimientos que consideran necesarios para algún trabajo concreto:

Porque por un lado sí, ya que te enseña a ser responsable, pero otro lado si quiero ser albañil la fotosíntesis no me sirve. (Alumno de $\mathrm{C}_{2}$ )

No se enseña algo laboral, todo es teórico en la secundaria. (Alumna de C2)

$\mathrm{Al}$ argumentar que la escuela no los está preparando adecuadamente para la inserción laboral, los/as jóvenes se basan en el criterio de la utilidad y aplicabilidad de los conocimientos, asociando dicha finalidad a una formación de carácter práctico e instrumental.

Considerando el conjunto de respuestas afirmativas y negativas, encontramos que los y las estudiantes coinciden al definir cómo sería una formación para el trabajo, haciendo referencia a la necesidad de trabajar conocimientos prácticos y adquirir ciertas actitudes y valores, pero difieren en cuanto a sus expectativas de inserción laboral.

En C2 y R2, como los alumnos/as aspiran a desempeñarse en puestos laborales que exigen mayor formación académica, el acceso al mundo del trabajo está subordinado a la posibilidad de realizar y concluir estudios superiores, mientras que en $\mathrm{C} 1$ y $\mathrm{R} 1$ 
los/as estudiantes no están considerando necesariamente la posibilidad de continuar estudiando para acceder a un mejor empleo. Advertimos, nuevamente, que la evaluación de la formación recibida está mediada por las expectativas a futuro, las que son influidas por las referencias familiares y las prácticas escolares.

\section{c) La preparación para el ejercicio de la ciudadanía democrática y la for- mación en valores para el desarrollo personal y la convivencia social}

Analizamos las respuestas en torno a la pregunta: “¿Crees que la educación que te ofrecen en la escuela te prepara de manera adecuada para la vida social en democracia, es decir, para ser un ciudadano/a responsable, consciente de tus derechos y obligaciones?".

El 75\% contestó que la escuela secundaria prepara adecuadamente, mientras que el $10 \%$ respondió que no y el 15\% marcó la opción no sabe o no contestó el ítem. En comparación a las otras dos finalidades, se advierte un mayor grado de conformidad respecto de la formación recibida. Sin embargo, los promedios ocultan diferencias entre establecimientos, como puede observarse en el siguiente gráfico:

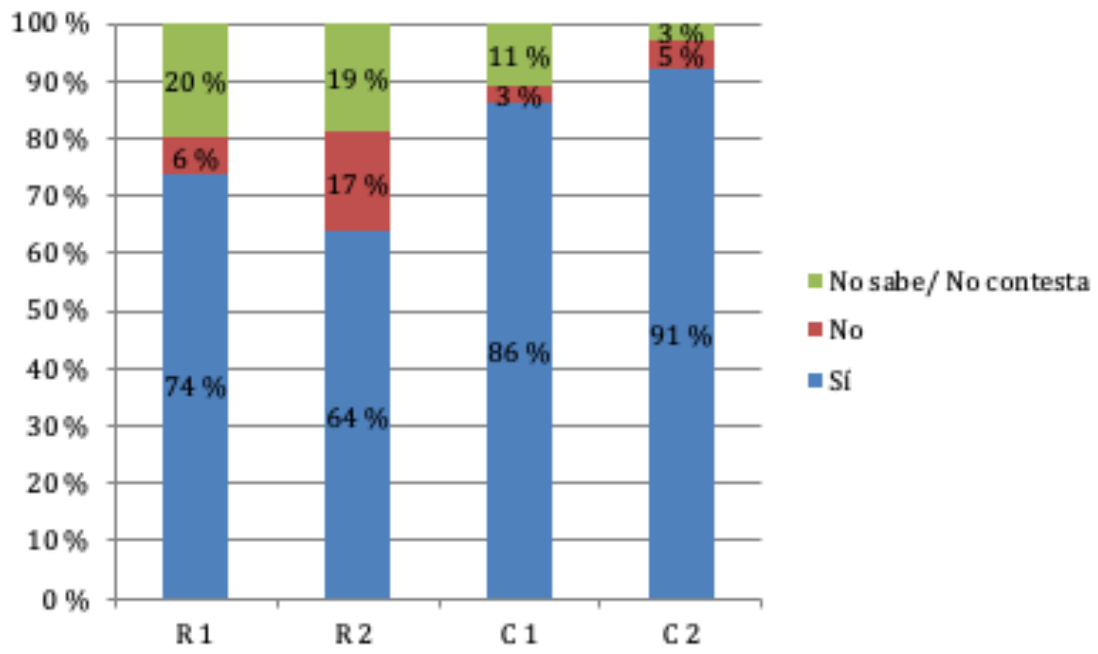

Gráfico 3. Distribución de los estudiantes por institución y si consideran que su establecimiento forma adecuadamente para el ejercicio de la ciudadanía

Se advierten diferencias en los porcentajes conforme a la ciudad de procedencia, siendo superiores en las escuelas de Corrientes que en las de Resistencia. Incluso en estas últimas hay una proporción significativa de estudiantes que no contestaron el ítem. Puede que estas diferencias se vinculen con características propias de la cultura chaqueña y correntina, aspectos en los que se podría profundizar en otro estudio.

En cuanto a las razones para justificar las respuestas afirmativas, estas tienden a valorizar el papel del establecimiento en la enseñanza de los derechos y obligaciones que tienen los/as estudiantes como ciudadanos/as, y en la transmisión de pautas de comportamiento y valores necesarios para la convivencia en sociedad:

Porque nos exigen un horario, una vestimenta, respetar y cumplir reglas. (Alumna de $\mathrm{C}_{1}$ )

Porque te enseñan a ser responsable y a cumplir tus obligaciones. (Alumno de C2)

Sí, porque nos enseña a cómo podemos ejercer nuestros derechos, a saber elegirlo que está bien o mal. (Alumno de $\mathrm{C}_{1}$ ) 
Con frecuencia los/as jóvenes aluden a un marco de reglas y comportamientos esperados, a una disciplina escolar, conformada por rutinas y hábitos. El conocimiento de derechos y obligaciones aparece integrado a cuestiones más generales relativas a la educación moral en valores, como el respeto y la responsabilidad.

En coincidencia con estas razones, los participantes de los grupos de discusión de C1 señalan que la responsabilidad, el respeto y el compañerismo son algunos de los aprendizajes más significativos que han adquirido en la escuela. Estas valoraciones se asocian al aprendizaje de actitudes y habilidades sociales para expresarse, compartir, convivir y relacionarse entre pares y con docentes y autoridades escolares. También perciben como aprendizajes relevantes la adquisición de hábitos, rutinas y de una disciplina para cumplir con las responsabilidades escolares. Encontramos similitudes con los hallazgos de Dussel, Brito y Núñez (2007), quienes identificaron un alto porcentaje de alumnos/as de diferentes niveles socioeconómicos que reconocen las relaciones o disposiciones sociales como los aprendizajes más importantes que ofrece la escuela, así como la formación en valores.

En el caso de estudiantes que sostienen que la escuela no los prepara adecuadamente para la ciudadanía democrática, en algunos casos, sostienen que ese tipo de formación no es responsabilidad de la escuela sino de las familias, porque "esas cosas se aprenden en la casa y no en la escuela" (Alumno de R2). En otros casos, plantean que la institución no posibilita el respeto por las normas de convivencia, pues "hay una serie de normas de la escuela que muchas veces no se cumplen" (Alumno de C2).

En general advertimos que los/as jóvenes asocian la educación para el ejercicio de la ciudadanía democrática con el aprendizaje de comportamientos adecuados al cumplimiento de normas y obligaciones, ponderando el aprendizaje de conductas y actitudes que permiten "tomar conciencia de lo que se debe hacer" y "saber elegir lo que está bien o mal".

Consideramos que estas concepciones aluden a un proceso de subjetivación en el cual el individuo se transforma en sujeto, adquiriendo un conocimiento de sí mismo y conformando intereses, opiniones y preferencias que definen "un modo de autogobierno, a la vez que se tornan gobernables" (Coicaud y Vidoz, 2015).

Se trata de visiones basadas en concepciones de ciudadanía asociadas a procesos de integración social, a diferencia de otras perspectivas que reconocen las tensiones y conflictos que devienen de las desigualdades sociales y de las distintas formas de estratificación y jerarquización social (Coicaud y Vidoz, 2015). Desde estas perspectivas la ciudadanía se define como un ámbito relacional, que alude a "una práctica conflictiva vinculada al poder, que refleja las luchas acerca de quiénes podrán decidir, sobre qué, al definir cuáles serán los problemas comunes y cómo serán abordados" (Jelin, 1993, citado por Coicaud y Vidoz, 2015:23).

En relación con este último enfoque, identificamos en las respuestas visiones alternativas y menos frecuentes, que aluden a espacios institucionales de participación, como el centro de estudiantes, valorados por los/as jóvenes como experiencias significativas para la construcción de ciudadanía democrática en el ámbito escolar:

Porque en la escuela hay derechos, como que los estudiantes tengan voz y voto. Contamos con Centro de Estudiantes, que es un paso previo a la vida democrática social que nos tocará vivir cuando deberemos elegir y sufragar en el futuro. (Alumna de $\mathrm{C}_{1}$ )

Desde esta concepción de ciudadanía, las escuelas se presentan como contextos de relaciones sociales complejas que incluyen la dimensión política de la convivencia 
entre jóvenes y adultos en el espacio escolar, en la que se juegan relaciones de poder, que inciden en las posibilidades de participación de los/as estudiantes en las decisiones colectivas. Pero, como dijimos, son las respuestas menos frecuentes, lo que nos hace pensar que las prácticas escolares cotidianas no promueven estas visiones alternativas.

\section{Conclusiones}

El análisis comparativo de percepciones y valoraciones sobre la formación recibida da cuenta de similitudes y diferencias entre las cuatro escuelas.

Entre las semejanzas, observamos que los niveles más altos de conformidad y consenso hacen referencia al aprendizaje de valores, actitudes y normas para la convivencia social y el ejercicio de la ciudadanía democrática. Los/as estudiantes comparten, en general, el modo en que entienden la formación para la ciudadanía, acorde a perspectivas que ponen el énfasis en la integración social.

En cuanto a la formación recibida para continuar estudios superiores, identificamos similitudes en los criterios de valoración de jóvenes de las cuatro escuelas que comparten la expectativa de continuar estudios superiores, especialmente universitarios. En sus fundamentos se advierte una mayor relevancia otorgada a los saberes teóricos, a procesos de enseñanza y aprendizaje para su apropiación y a la exigencia académica, como condiciones de una preparación adecuada para el futuro desempeño en una carrera universitaria.

Estos criterios de valoración difieren de los manifestados por otros/as jóvenes de C1 y R1, quienes se centran en la orientación y el estímulo brindado por los docentes. En esos casos, aparece con mayor énfasis la idea del acceso a la educación superior como condición para acceder a mejores trabajos, y la escuela secundaria resulta significada como oportunidad de progreso social. Como posible explicación de estas diferencias en las percepciones, valoraciones y expectativas estudiantiles, y en concordancia con las perspectivas teóricas que asumimos para definir el concepto de experiencia escolar, consideramos, por un lado, la influencia de las prácticas formativas que desarrollan los establecimientos, y por el otro,las representaciones y expectativas familiares sobre los estudios superiores.

Asimismo, identificamos criterios de valoración compartidos acerca del tipo de contenidos y prácticas pedagógicas requeridos para una adecuada formación para el trabajo. Los y las estudiantes señalan la necesidad de trabajar conocimientos prácticos y adquirir ciertas actitudes y valores, pero difieren en cuanto a sus expectativas de inserción laboral, dependiendo de ellas cuánto valor le asignan a la continuación de estudios superiores y a la importancia del trabajo con el conocimiento disciplinar y la exigencia académica. También en lo atinente a esta finalidad, la evaluación de la formación recibida está mediada por las expectativas a futuro.

De este modo, el análisis realizado introduce elementos referidos a las relaciones entre sentidos atribuidos a la escuela y la construcción de proyectos de vida futuros, los cuales resultanasociados a lasposiciones educativas y laborales anheladas, así como tambiénlos vínculos entre los sentidos de la experiencia escolar, las prácticas escolares que las configuran y los diferentes "puntos de partida" de los y las estudiantes definidos por sus condiciones socioeconómicas de origen. 


\section{Q Bibliografía}

》 Andrade, L. (2002). Construcción social e individual de significados: aportes para su comprensión. Estudios Sociológicos, 20(1),pp.199-320.

» Coicaud, S. y Vidoz, S. (2015). Aportes para pensar la ciudadanía. En Coicaud, S. (Ed.),La educación en ciudadanía como construcción de subjetividad política. Jóvenes, escuela y proyectos. Buenos Aires: Miño y Dávila.

»Dubet, F. y Martuccelli, D. (1998). En la escuela. Sociología de la experiencia escolar. España: Losada.

»Dussel, I., Brito, A. y Núñez, P. (2007). Más allá de la crisis: visión de alumnos y profesores de la escuela secundaria argentina. Buenos Aires: Santillana.

»Contreras, J. y Pérez de Lara Ferré, N. (Comps.) (2010). Investigar la experiencia educativa. Madrid: Morata.

» Guzmán Gómez, C. ySaucedo Ramos, C. (2015). Experiencias, vivencias y sentidos en torno a la escuela y a los estudios. Abordajes desde la perspectiva de alumnos y estudiantes. Revista Mexicana de Investigación Educativa, 20 (67),pp.1019-1054.

»Larrosa, J. (2004). La Experiencia de la lectura. Estudios sobre Literatura y Formación. México: FCE.

»Llinás, P. (2009). Imágenes y sentidos de la experiencia escolar: percepciones de los estudiantes sobre la escuela secundaria. Propuesta Educativa (32),pp.95-104.

» Llomovatte, S. y Kaplan, C. (Coords.) (2012). Desigualdad educativa. La naturaleza como pretexto. Buenos Aires: Novedades Educativas.

» Maddonni, P. (2014). El estigma del fracaso escolar: nuevos formatos para la inclusión y la democratización de la educación. Buenos Aires: Paidós.

» Maldonado, M.; Servetto, S. y Molina, G. (2015). Experiencias juveniles en escuela secundaria: avances y desafíos de un estudio socio-antropológico. En Pinkasz, D. (Comp.), La investigación sobre educación secundaria en la Argentina en la última década. Buenos Aires: FLACSO.

" Montesinos, M. P.; Sinisi, L. y Schoo, S. (2009). Sentidos en torno a la "obligatoriedad" de la educación secundaria. Buenos Aires: Dirección Nacional de Información y Evaluación de la Calidad Educativa, Ministerio de Educación de la Nación.

» Rockwell, E. (Coord.) (1995).La escuela cotidiana. México: Fondo de Cultura Económica.

» Rockwell, E. (2006).Los niños en los intersticios de la cotidianeidad escolar: żresistencia, apropiación o subversión? (Conferencia). XI Simposio Interamericano de Etnografía de la Educación. Buenos Aires, Argentina.

» Tiramonti, G. (2011). Variaciones sobre la forma escolar: límites y posibilidades de la escuela media. Rosario: Homo Sapiens.

» Tiramonti, G. y Montes, N. (2009). La escuela media en debate: Problemas actuales y perspectivas desde la investigación. Buenos Aires: Manantial. 


\section{Patricia Malena Delgado}

Magister en Docencia Universitaria, Universidad Nacional del Nordeste. Profesora Adjunta, Departamento de Ciencias de la Educación. Directora del Instituto de Investigaciones en Educación, Facultad de Humanidades, Universidad Nacional del Nordeste, Argentina. patridelgadow@gmail.com

\section{Cinthia Denise Amud}

Doctoranda en Ciencias de la Educación,Universidad Nacional de Córdoba. Licenciada en Ciencias de la Educación, Universidad Nacional del Nordeste. Departamento de Ciencias de la Educación, Universidad Nacional del Nordeste, Argentina. deniamud@ gmail.com

\section{Jorge Samuel Salas}

Profesor en Ciencias de la Educación, Universidad Nacional del Nordeste. Becario de Iniciación, Secretaría General de Ciencia y Técnica de la Universidad Nacional del Nordeste, Argentina.jorgesamuelsalas@gmail.com 
